\title{
Researches on damage identification in passive vibro-isolation devices
}

\author{
A. Leopa*, S. Nastac and C. Debeleac \\ Research Centre for Mechanics of Machines and Technological Equipments, "Dunarea de Jos" University, Braila, \\ Romania
}

\begin{abstract}
This paper deals with the theoretical aspects combined with experimental analysis regarding early damage identification in passive vibro-isolation devices. Basically, this research presents the relevant results obtained for a singular element. Rubber based on elements is discussed especially. This study was a naturally fall-back of the authors large analysis regarding the dynamic behaviour of the passive isolation devices against vibration, shocks and seismic waves. Main hypothesis supposed that on the exploitation time, all technical devices and systems acquire different levels of wearing because of the dynamic overloads and their derivative influences (aging, fatigue, energy dissipation, external heating or cooling, etc.). Hereby the performance characteristics changes and the system becomes working improperly. Numerical simulations were developed for simple spatial configuration of isolation device. Stochastic approach of essential results was briefly presented nearby the relevant results and discussions.
\end{abstract}

Keywords: Damage, identification, vibration, isolation, rubber

\section{Introduction}

For vibro-isolation devices, during the behaviour evaluation and measurements, both in the laboratory and "in situ" tests, it was observed that the damages level grows up with the effective exploitation time, and especially appears on the visco-elastic kernel. Taking into account the final influence of visco-elastic elements on the global isolation degree of the insulated system, it results the basic idea of damage level evaluation based on the transfer characteristics of the dynamic system. The main advantages of this method are: non-destructive method, increased safety, reduced costs. Another advantage of this method is the possibility of evaluation as a continuous process, and the possibility of detection of the beginning of important failures before these already acquire a critical level and disturb the dynamics and the integrity of the entire isolation system.

This study continues a series of past theoretical and practical analysis regarding passive vibro-isolation performances, methods, maintenance, monitoring, and damage detection [5-17]. The additional gain of this research is the estimation of primary sources for vibro-isolation elements degradation and, based on this, a serviceable way for monitoring and damaged area detection in real time.

Like many other technical systems, the vibro-isolation elements have a theoretical lifetime which, in practice, presents more or less diminution. Intensive random dynamic loads can produce dangerous damages and a fast degradation. Aging of the base core material induces slow, but irremediable degradation of the element.

Aging of the rubber from the visco-elastic element core can have many causes. One of the most important is the internal energy dissipation during the working cycle. Internal heat leads to major changes of the material characteristics. A major decrease of the performance level can occur. One part of the studied elements had only local and temporary effects but the other acquire permanent changes.

*Corresponding author: A. Leopa, Research Centre for Mechanics of Machines and Technological Equipments, "Dunarea de Jos" University, Calea Calarasilor 29, 810017, Braila, Romania. E-mail: adrian.leopa@ugal.ro. 
Note that this analysis procedure is framed into the Structural Health Monitoring Concept, which enables Conditions - Based Maintenance at structures through diagnosis of the current health status during exploitation [5]. Parts of the theoretical approaches, that enable the method implementation, are based on Reverse Engineering concept and Inverse Problems theory [3].

\section{Theoretical basics}

First of all, the linkage between the material characteristics changes and the natural frequency shifting has to be shown. According to the inverse problem theory with applications in vibration analysis [3], and taking into consideration the initial hypothesis of discrete structural composition of the vibratory isolated systems, the direct involvement of the rigidity variance on natural frequencies spectral degeneration can be demonstrated.

Supposing the stiffness and the mass matrices denoted by $\mathbf{K}$ and $\mathbf{M}$, the mode shape vector by $\mathbf{x}$, the squared natural frequencies matrix by $\Omega$, and the changes of these parameters such as

$$
\begin{array}{cc}
\mathbf{K}+\Delta \mathbf{K} ; & \mathbf{M}+\Delta \mathbf{M} \\
\boldsymbol{\Omega}+\Delta \boldsymbol{\Omega} ; & \mathbf{x}+\Delta \mathbf{x}
\end{array}
$$

and the general discrete equation as

$$
(\mathbf{K}-\mathbf{\Omega M}) \mathbf{x}=\mathbf{0},
$$

the replacement of changed main parameters (1) in (2) leads to an extended equation. Using the basic hypothesis that all the terms of second order and more may be ignored, supposing the null changes of masses, multiplying with transposed mass-normalized solution $\mathbf{x}$ and making the computations result in

$$
\Delta \boldsymbol{\Omega}=\mathbf{x}^{T} \Delta \mathbf{K} \mathbf{x}
$$

Equation (3) shows that the shifting of natural frequencies can be found when the rigidity changes are known. Also, Eq. (3) reveals the possibility of identification of which elements lead to the specified demotion in stiffness. Using this method, the linkage between damping changes and natural frequency shifting can also be obtained.

One of the sources for degrading of the rubber core of vibro-isolation elements is damping energy [12]. Dissipated energy for each working time leads to internal heat emergence which are transmitted from inside to outside the active part of the vibro-isolation element. This process includes the entire volume of rubber element. The environment temperature on the working place is also an important factor for this process. The main characteristics of the material which acquire changes that can affect the global performances are the rigidity and the damping. The intensive and various loads that actuate the isolators produce two basic types of effects, namely: temporary effects which modify the characteristics only during the working cycle and the element recovers the initial state after that, and the permanent effects - which appear because of the cumulative process of degradation and the element recovers partially the initial state after the external excitation stops.

The basic rheological model used in this research to simulate the rubber vibro-isolation element is depicted in Fig. 1. According to past researches [4,12], the complex model framed by Voigt and Maxwell bodies was supposed. Using complex domain formulations and Laplace's direct and inverse operators results the global characteristic equation of the model in Fig. 1. Hereby, the complex formulations are respectively in the frequency domain

$$
F(j \omega)=\left(k_{2}+\frac{\omega^{2} c_{1}^{2} k_{1}}{k_{1}^{2}+\omega^{2} c_{1}^{2}}\right) X(j \omega)+\left(c_{2}+\frac{k_{1}^{2} c_{1}}{k_{1}^{2}+\omega^{2} c_{1}^{2}}\right) j \omega X(j \omega)
$$

and in the time domain 


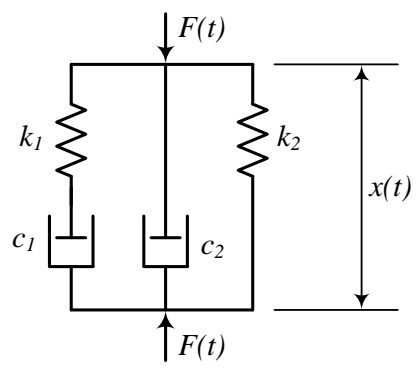

Fig. 1. Basic rheological model of the passive vibro-isolation elements (see text for details).

$$
f(t)=\left(k_{2}+\frac{\omega^{2} c_{1}^{2} k_{1}}{k_{1}^{2}+\omega^{2} c_{1}^{2}}\right) x(t)+\left(c_{2}+\frac{k_{1}^{2} c_{1}}{k_{1}^{2}+\omega^{2} c_{1}^{2}}\right) \frac{d x(t)}{d t}
$$

where $j=\sqrt{-1}, k_{i}$ denotes stiffness coefficients and $c_{i}$ denotes damping coefficients, $\omega$ means pulsation of external signal, $f(t)$ denotes the external force and $x(t)$ the deformation applied to the model.

These formulations have the advantage that they provide a simple expression such as the Voigt classical model, which mainly means easiness for analytical approaches. While Eqs (4) and (5) provide a very convenient way to analyze the element behaviour, the authors consider that it is necessary to evaluate the general constitutive equation of this model so that the evolution of the model supposing different types of external signals can be computed. Hereby, the constitutive equation is

$$
f(t)=\left[\frac{k_{1} c_{1} D}{k_{1}+c_{1} D}+k_{2}+c_{2} D\right] x(t)
$$

where the notation $D=\frac{\partial}{\partial t}$ was used. Generally, the stiffness $k_{i}$ and damping $c_{i}$ coefficients have proportional values so that Eq. (6) becomes

$$
f(t)=\left[\frac{k_{1} c_{1} D}{k_{1}+c_{1} D}+\lambda_{k} k_{1}+\lambda_{c} c_{1} D\right] x(t)
$$

or

$$
f(t)=\left[\frac{\lambda_{k} k_{1}^{2}+\left(1+\lambda_{k}+\lambda_{c}\right) k_{1} c_{1} D+\lambda_{c} c_{1}^{2} D^{2}}{k_{1}+c_{1} D}\right] x(t)
$$

In particular, one of the two variables has to be known from the initial conditions. Thus, Eq. (8) becomes a 1st or 2nd order differential equation which can be easily solved. It results the specific evolution of the stress or of the deformation with respect to the provided restriction. Knowing the input and the output of this system - such as $f(t)$ and $x(t)$, the transfer characteristic of the element can be evaluated and further both the foresight behaviour, and the deviation from the natural evolution.

\section{Results and discussions}

Figure 2 shows a few types of passive vibro-isolation elements based on synthetic rubber. These elements were designed for compression charging and can be used both as a single element (see Figs $2 \mathrm{a}-\mathrm{c}$ ), and as a complex cartridge device supposing a certain number of identical elements parallelly mounted (see Fig. 2d). 
Experimental tests and numerical simulations [5-12], developed for a rubber vibro-isolation element (Fig. 2c) with dimensions $150 \times 150 \times 150 \mathrm{~mm}$, loaded with $500 \mathrm{~N}$, showed that the internal temperature, for a stabilized regime and common conditions, can get around $95^{\circ} \mathrm{C}$ into the core and $70^{\circ} \mathrm{C}$ on the external surfaces. The time interval for thermal and functional stabilization was 700-800 s. In case of dangerous evolution, the temperature may rise over $200^{\circ} \mathrm{C}$ in only $20-30 \mathrm{~s}$. Also, the amplitude of the motion increases very much (10 to 11 times). In this case, the element can be destroyed.

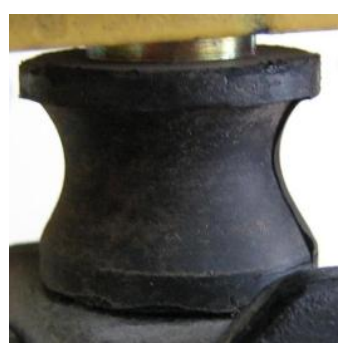

(a)

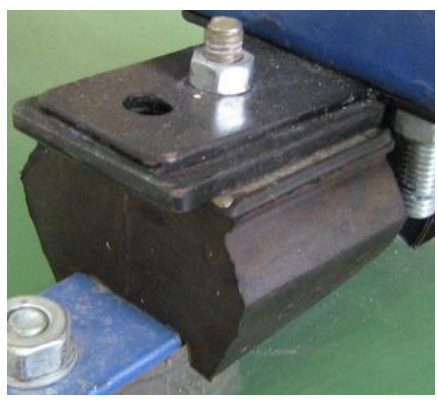

(c)

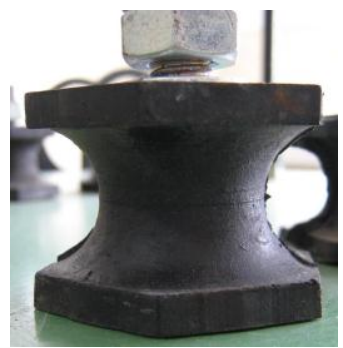

(b)

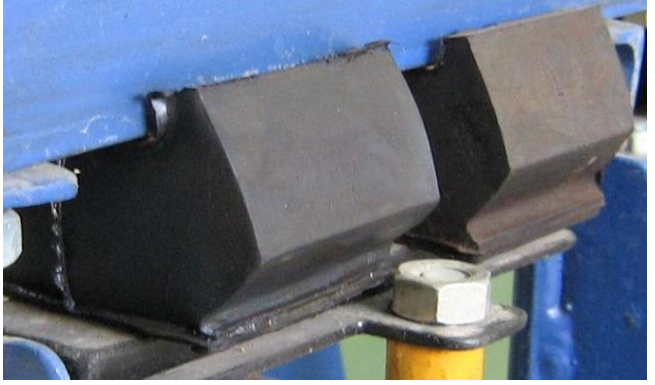

(d)

Fig. 2. A few types of passive vibro-isolation elements (see text for details).

For estimation of the main parameters change during the exploitation time, an exponential law was used for rigidity, respectively damping, in accordance with the internal temperature obtained as a direct result of the mechanical energy dissipation into the entire volume of the element. Hereby, the proposed expression for rigidity dependence with temperature is

$$
k=k_{o}\left[\alpha_{k}+\beta_{k} e^{\left[\delta_{k}\left(t_{r e f}-t_{i}\right)\right]}\right]
$$

and the expression for the damping coefficient is

$$
c=c_{o}\left[\alpha_{c}+\beta_{c} e^{\left[\delta_{c}\left(t_{\text {ref }}-t_{i}\right)\right]}\right]
$$

where $\alpha, \beta, \delta$ denote thermal changes parameters depending on the shape and material characteristics, $k_{o}$ and $c_{o}$ means the rigidity and damping at reference temperature, $t_{r e f}$ is the reference temperature (which usually is $20^{\circ} \mathrm{C}$ ) and $t_{i}$ is the instantaneous temperature at the evaluated point inside the vibro-isolation element. Hereby, in Fig. 3 is depicted the evolution of the transmissibility with respect to the internal temperature and external excitation. A relative stabilization of the natural frequency can observed for temperature values around $80^{\circ} \mathrm{C}$, but with a permanent increase of the magnitude in the resonance area. From this diagram, it results that post-resonance working regime can assure a stable dynamic evolution because of the resonance frequency decrease with the increase of the temperature. For the same reason, a dangerous evolution can happen for pre-resonant working state. 


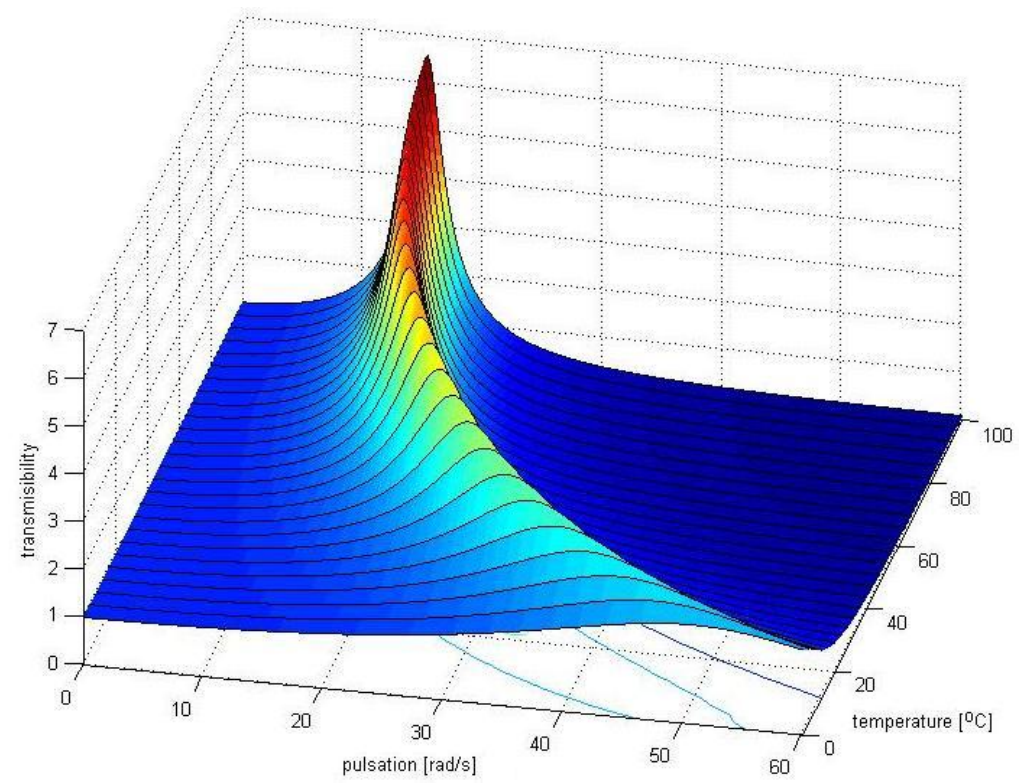

Fig. 3. Transmissibility evolution with pulsation and temperature changing.

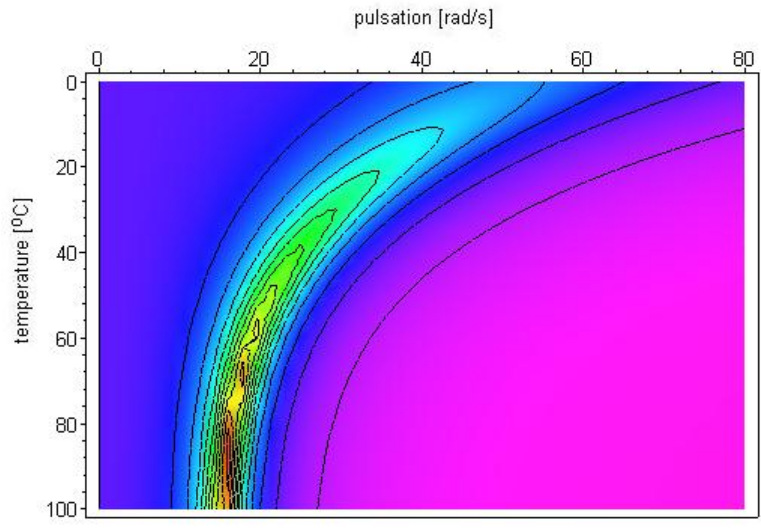

(a)

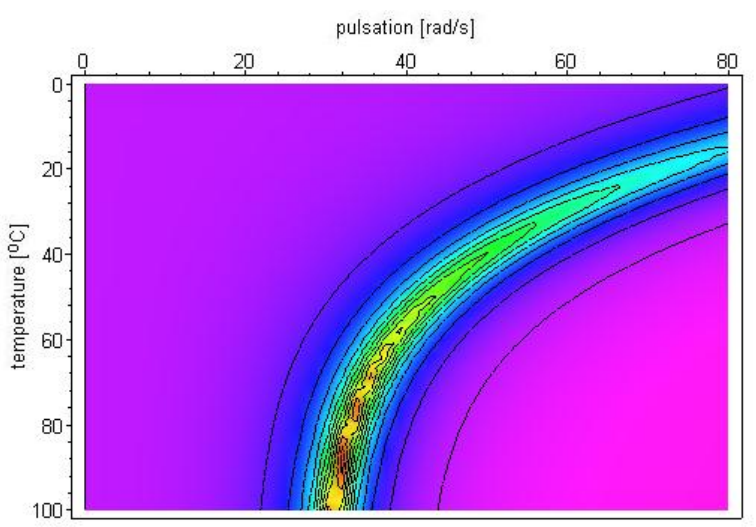

(b)

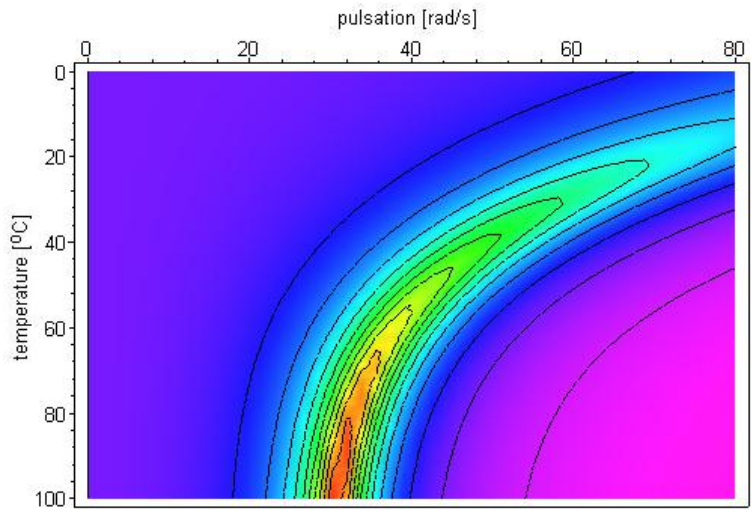

(c)

Fig. 4. Transmissibility evolution vs. rigidity and damping permanent changes (first case of analysis). 
In Fig. 4 are depicted three diagrams which show what is happening during a long intensive exploitation time. Hereby, the cumulative thermal effects inside the rubber element lead to permanent changes of characteristics. This is indicated through rigidity increase, with constant damping (see Fig. 4b) and both rigidity and damping growing up (see Fig. 4c). In Fig. 4a is depicted the initial case, without any perm

anent changes. Comparative analysis of the three diagrams reveals a consistent and continuous diminution of post-resonant safety working area. The lower limit of excitation pulsation for post-resonant non-dangerous working regime has to be increased in this case. Quantitatively, the transmissibility has the following maximum values: 7 for the case in Fig. 4a; 13 for the case in Fig. 4b; 6.5 for the case in Fig. 4c.

The diagrams presented in Fig. 5 show the transmissibility evolution for the cases of rigidity increasing with aging, but supposing a decrease in damping. Hereby, in Fig. 5a the rigidity has the initial reference value and the damping decreases at half value of the initial reference value. Otherwise, in Fig. $5 b$ the rigidity is growing up four times and the damping remains at half value of the reference value. In comparison with the diagrams in Fig. 4 when rigidity is growing up four times and damping two times, the transmissibility reaches the following maximum values: 12 for the case in Fig. 5a and 24 for the case in Fig. 5b.

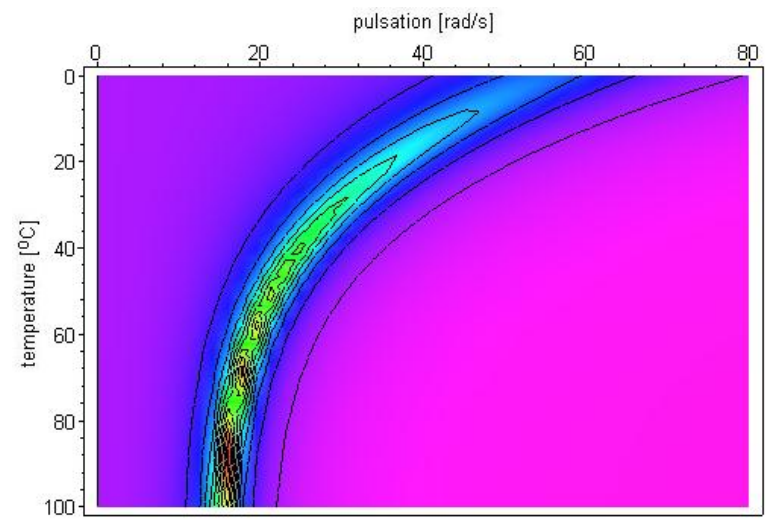

(a)

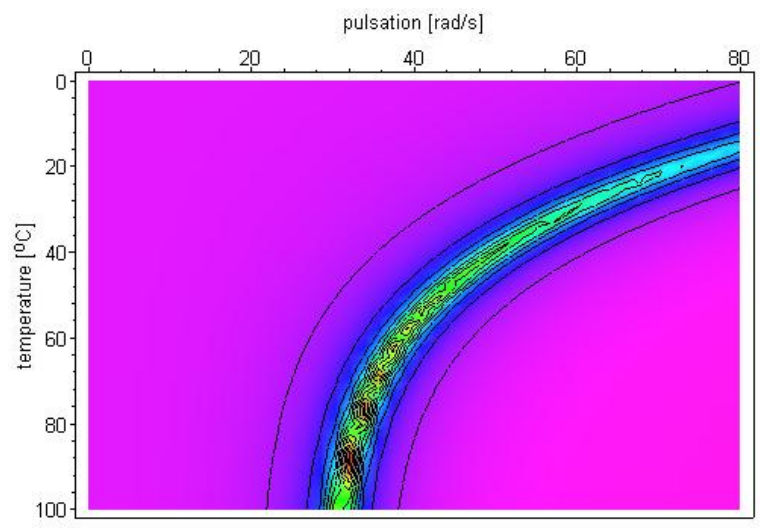

(b)

Fig. 5. Transmissibility evolution vs. rigidity and damping permanent changes (second case of analysis).

The case of rigidity decreasing was not presented in this paper. In that case, the low reference value of rigidity provides an initial shifting to the left side of the natural frequency. During the exploitation and due to the thermal additional shifting for natural frequency (also to the left side, at low values), the necessary post-resonant working conditions was assured whatever the values of the external excitation frequency are.

\section{Conclusions}

Even if the evolution under external dynamic loads was evaluated for rubber vibro-isolation elements in case of basic material aging, it is very difficult to accurately estimate the current value at each moment of time for the entire set of the characteristic parameters. Simulations on virtual model allowed to determine the appropriate behaviour according to the instrumental tests for each rubber element. But for differential aging of the rubber core of the isolator, only a virtual analysis was developed taking into account the real difficulties in the measurement of the core instantaneous temperature. In this case, an exponential law was adopted, such as in Eqs (9) and (10).

As a final concluding remark, it has to be mentioned that the evolution laws for aging have to take into account the hardening and/or softening of both the stiffness, and the dissipation. Hereby the hardening law of rigidity is dangerous both for the rubber element and, of course, for the entire insulated structure because of the diminution of the cut-off frequency of the isolator. The reduction of the post-resonance area is also a dangerous phenomenon which has to be avoided in practice. 
The future research will finalize the instrumental evaluation of rubber core degrading during the exploitation time and try to estimate a set of mathematical laws which can simulate the aging process.

\section{Acknowledgments}

This work was supported by UEFISCDI (formerly CNCSIS-UEFISCSU) project number PN II-RU-PD code $597 / 2010$.

\section{References}

[1] G. Axinti, Compendiu de mecanica (Mechanics Digest), Editura UTM, Chisinau, 2008.

[2] P. Bratu, Vibratiile sistemelor elastice (Elastic Systems Vibrations), Editura Tehnica, Bucuresti, 2000.

[2] P. Bratu, Mecanica teoretica (Theoretical Mechanics), Editura Impuls, Bucuresti, 2006.

[3] G.M.L. Gladwell, Inverse Problems in Vibration, Kluwer Academic Publishers, Dordrecht, 2004.

[4] L. Grigorescu, Gh. Oproescu and S. Nastac, Teoria sistemelor si automatizari (Theory of Systems and Automatizations), Editura Impuls, Bucuresti, 2002.

[5] T. Johnson, D. Adams and M. Schiefer, An Analitical and Experimental Study to Assess Structural Damage and Integrity Using Dynamic Transmissibility, The Proceedings of the 20th International Modal Analysis Conference, 2002.

[6] S. Nastac, Advances on Characteristics Changing and Working State Degrading of Passive Vibroisolation Elements, Plenary lecture, The 5th IASME International Conference on Continuum Mechanics (CM'10), February 23-25, 2010, University of Cambridge, UK.

[7] S. Nastac, G.R. Gillich, A. Leopa and C. Debeleac, Spectral Degeneration At Damaged Vibroisolation Systems, The Proceedings of The Annual Symposium of the Institute of Solid Mechanics SISOM 2009 and Session of the Commission of Acoustics, Commission of Acoustics of Romanian Academy, Bucharest, Romania, 2009.

[8] S. Nastac, Analysis of Working Performances at Damaged Vibration Isolation Devices, in: Annals of DAAAM for 2008 \& Proceedings of the 19th International DAAAM Symposium, B. Katalinic, ed., ISBN 978-3-901509-68-1, ISSN 1726-9679, Published by DAAAM International, Vienna, Austria, 2008.

[9] S. Nastac, Performances Evaluation at Damaged Vibration Isolation Devices, Chapter 47 in: DAAAM International Scientific Book 2008, B. Katalinic, ed., Published by DAAAM International, Vienna, Austria, 2008, pp. 551-564.

[10] S. Nastac and Gh. Oproescu, About Damages Identification Methods at MDOF Dynamic Systems, The Proceedings of the Annual Symposium of the Institute of Solid Mechanics SISOM 2008 and Session of the Commission of Acoustics, Romanian Academy, Department of Technical Sciences, Bucharest, Romania, 2008

[11] S. Nastac, The Diminishing of the Working Performances at the Demotion Antivibrational Systems, The Proceedings of the IX-th Symposium Acoustics and Vibration of Mechanical Structures - AVMS 2007, Romanian Academy, Timisoara, Romania, 2007, pp. 206-210.

[12] Gh. Oproescu and C. Debeleac, Classic and Modern in the Simulation of the Dynamic Behavior of the Technical Systems, in: Plenary invited paper at Exploratory Workshop - Advanced Rheological Modelling with Micro- and Macro-structural Concept of Neoprene Composites for Base Isolation against Shocks and Vibrations, Braila, Romania, 2008, published in proceedings, Ed. Impuls, ISBN 978-973-8132-68-9.

[13] L. Pesek, L. Pust and P. Sulc, FEM Modeling of Thermo-mechanical Interaction in Pre-pressed Rubber Block, Engineering Mechanics 14(1/2) (2007), 3-11.

[14] L. Pesek, F. Vanek, P. Prochazka, V. Bula and J. Cibulka, New test approach and evaluation of dynamic and thermal parameters of elastomers, Engineering Mechanics 16(3) (2009), 97-207.

[15] A.G. Piersol and T.L. Paez, Harris'Shock and Vibration Handbook, Sixth Edition, The McGraw-Hill Companies, 2010

[16] G. Ramorino, D. Vetturi, D. Cambiaghi, A. Pegoretti and T. Ricco, Developments in dynamic testing of rubber compounds: Assessment of non-linear effects, Polymer Testing 22 (2003), 681-687.

[17] A.F. Vakakis, Advanced nonlinear strategies for vibration mitigation and system identification, CISM Courses and Lectures 518, SpringerWienNewYork, 2010. 

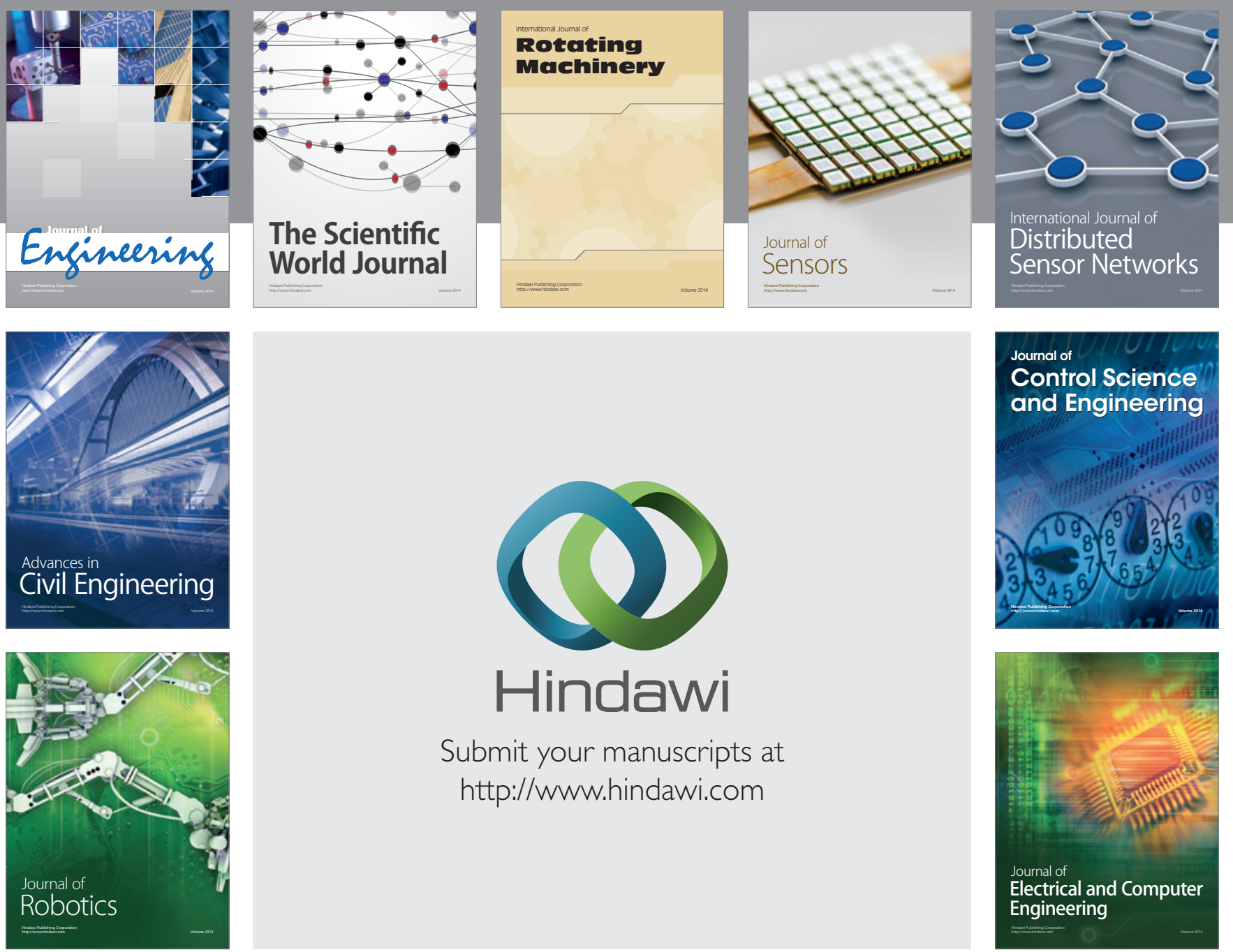

Submit your manuscripts at

http://www.hindawi.com
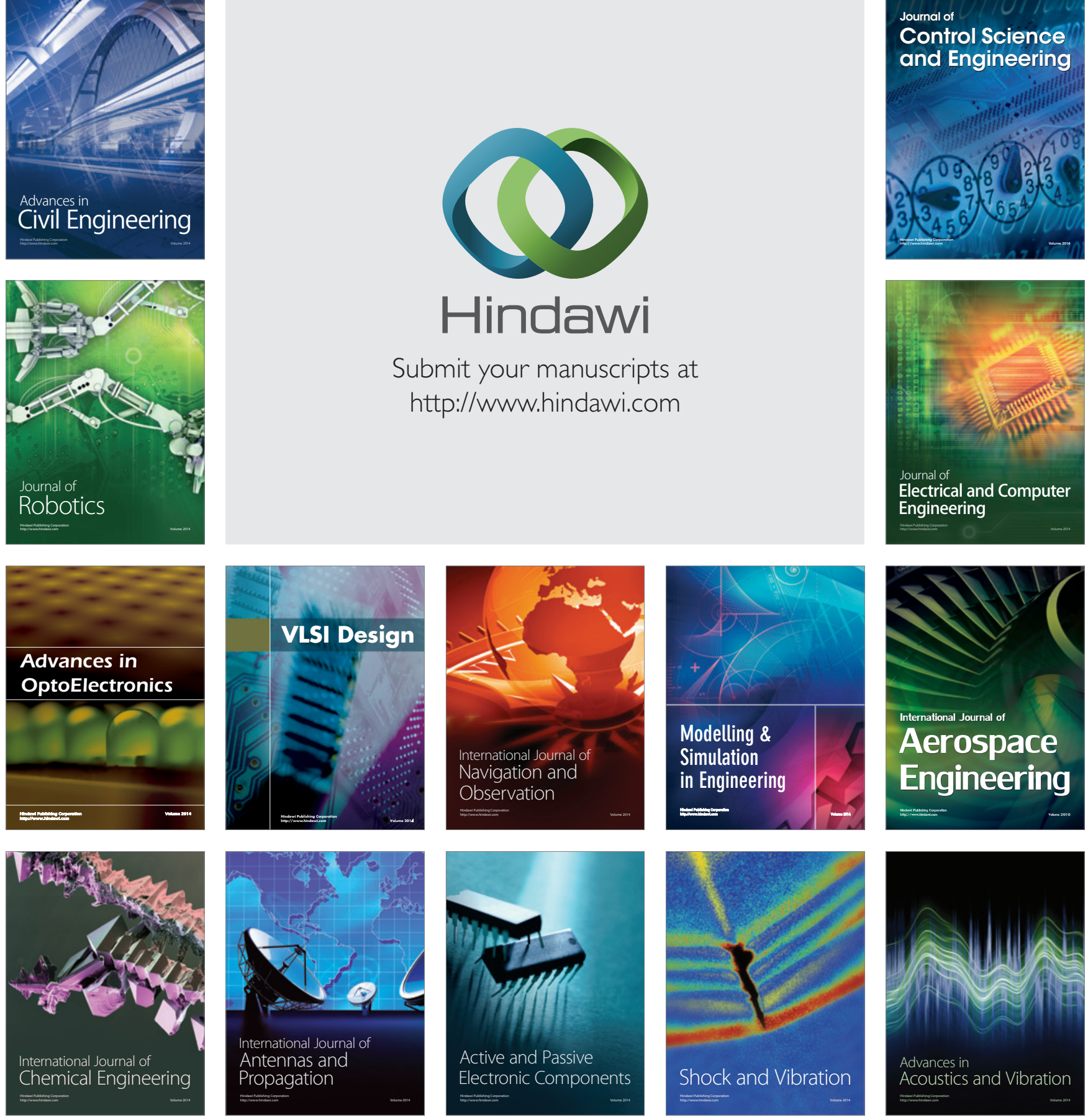